\title{
Correction to: Role of peptaibols and lytic enzymes of Trichoderma cerinum Gur1 in biocontrol of Fusarium oxysporum and chickpea wilt
}

\author{
Ekta Khare ${ }^{1}$ (D) Sanoj Kumar ${ }^{1} \cdot{\text { Kangmin } \mathrm{Kim}^{2}}^{2}$
}

Published online: 13 August 2018

c) Society for Environmental Sustainability 2018

\section{Correction to: Environmental Sustainability (2018) 1:39-47 https://doi.org/10.1007/s42398-018-0001-7}

The original article was published with the wrong title. The correct title is: Role of peptaibols and lytic enzymes of Trichoderma cerinum Gur1 in biocontrol of Fusarium oxysporum and chickpea wilt. s42398-018-0001-7.

Ekta Khare

ekhare.cnu@gmail.com

1 Department of Microbiology, Institute of Biosciences and Biotechnology, Chhatrapati Shahu Ji Maharaj University, Kanpur, UP 208024, India

2 Division of Biotechnology, SELS Institute, College of Environmental and Bioresource Sciences, Chonbuk National University, Iksan 54596, Republic of Korea 